\title{
APTIDÃO FÍSICA E ANTROPOMETRIA EM IDOSOS PRATICANTES DE PRÁTICAS CORPORAIS
}

\begin{abstract}
Airton Spillere Dalmagro Junior Acadêmico do Curso de Educação Física, Universidade do Extremo Sul Catarinense, airtonsdj@gmail.com Luana Cademartori Minghelli Profissional de Educação Física, Universidade do Extremo Sul Catarinense, lua_minghelli@yahoo.com.br Sabrina Furtunato de Ávila Profissional de Educação Física, Universidade do Extremo Sul Catarinense, sabrinafavila@yahoo.com.br Amanda Biava Lima Acadêmica do Curso de Educação Física, Universidade do Extremo Sul Catarinense, amanda_biava@hotmail.com Francine Costa de Bom Mestra, Universidade do Extremo Sul Catarinense, costafrancine@hotmail.com Joni Marcio de Farias Doutor, Universidade do Extremo Sul Catarinense, jmf@unesc.net Ana Maria Volpato Doutora, Universidade do Extremo Sul Catarinense, volpatoam@gmail.com Victor Julierme Santos da Conceiçáo Doutor, Universidade Federal de Santa Catarina, victorjulierme@gmail.com
\end{abstract}




\section{RESUMO}

Envelhecimento pode ser entendido como um processo dinâmico, progressivo e irreversível. Esse processo gera diversas alteraçóes no indivíduo, tanto biológicas quanto psicológicas e sociais, comprometendo a sua capacidade de responder ao estresse ambiental e à manutenção da homeostasia (MATSUDO, 2000; SHEPHARD, 2003). Tais alteraçôes refletem na diminuição das capacidades físicas e na composição corporal. O objetivo desse estudo é investigar a efetividade de práticas corporais sobre a aptidão física e antropometria em idosos. Participaram das avaliações e das práticas corporais um total de 92 idosos (71 homens e 21 mulheres), com idade entre 60 e 75 anos, participantes do Programa Multidisciplinar de Atenção à Saúde do Idoso, realizado na Universidade do Extremo Sul Catarinense (UNESC). Antes e após os 8 meses de estudo, foram avaliadas as seguintes variáveis: massa corporal, índice de massa corporal (IMC), circunferência da cintura e quadril, relação cintura quadril (RCQ), flexibilidade e força de membros superiores. A análise estatística foi feita por teste de variância de duas vias (ANOVA two-way). O nível de significância admitido foi de $\mathrm{p}<$ 0,05 . As análises indicaram massa corporal $(\mathrm{p}<0,0001)$ e circunferência da cintura $(\mathrm{p}=0,0004)$ maiores para os homens, em relação às mulheres, nos dois períodos analisados. Os níveis de flexibilidade apresentaram valores maiores para as mulheres, em relação aos homens $(\mathrm{p}=0.00021)$. Valores de IMC, circunferência do quadril, RCQ e força mostraram-se semelhantes. Os resultados significantes encontrados ressaltam as diferenças observadas entre os gêneros, ao longo do processo de envelhecimento de homens e de mulheres. Além disso, as práticas corporais foram efetivas ao manter os níveis das capacidades físicas e variáveis antropométricas observadas, ressaltando a importância da inserção dessas atividades em idosos para a manutenção da autonomia e independência.

Palavras-chave: Envelhecimento; Aptidão Física; Práticas Corporais. 


\section{REFERÊNCIAS}

MATSUDO, S. M.; MATSUDO, V. K. R.; BARROS NETO, T. L. Impacto do envelhecimento nas variáveis antropométricas, neuromotoras e metabólicas da aptidão física. Revista Brasileira de Ciência e Movimento, Brasília, v.8, n.4, p.21-32, 2000.

SHEPHARD, R. J. Envelhecimento, atividade física e saúde. São Paulo: Phorte, 2003. 